Maria Chudnovsky $\cdot$ Neil Robertson $\cdot$ P. D. Seymour $\cdot$ Robin Thomas

\title{
Progress on Perfect Graphs
}

Received: December 19, 2002 / Accepted: April 29, 2003

\begin{abstract}
A graph is perfect if for every induced subgraph, the chromatic number is equal to the maximum size of a complete subgraph. The class of perfect graphs is important for several reasons. For instance, many problems of interest in practice but intractable in general can be solved efficiently when restricted to the class of perfect graphs. Also, the question of when a certain class of linear programs always have an integer solution can be answered in terms of perfection of an associated graph.

In the first part of the paper we survey the main aspects of perfect graphs and their relevance. In the second part we outline our recent proof of the Strong Perfect Graph Conjecture of Berge from 1961, the following: a graph is perfect if and only if it has no induced subgraph isomorphic to an odd cycle of length at least five, or the complement of such an odd cycle.
\end{abstract}

Key words. Berge graph, perfect graph, skew partition

\section{Introduction}

In this paper graphs are finite and simple; that is, they have no loops or multiple edges. Let $G$ be a graph. A hole in $G$ is an induced cycle of length at least four. An antihole in $G$ is an induced subgraph isomorphic to the complement of a hole. (The complement of a graph $G$ is the graph with vertex-set $V(G)$ and edge-set consisting precisely of all distinct pairs of vertices that are not adjacent in $G$.) A clique in $G$ is a set $X \subseteq V(G)$ of pairwise adjacent vertices, and a stable set is a set of pairwise non-adjacent vertices. The size of a maximum clique of a graph $G$ is denoted by $\omega(G)$ and the size of a maximum stable set is denoted by $\alpha(G)$. The chromatic number of $G$, denoted by $\chi(G)$, is the least number of colors needed to color the vertices of $G$ in such a way that adjacent vertices receive different colors. In other words, $\chi(G)$ is the minimum number $k$ such that the vertex-set $V(G)$ of $G$ can be partitioned into $k$ stable sets. Clearly $\chi(G) \geq \omega(G)$ for every graph $G$, but equality need not hold. For instance, if $G$ is an odd hole (i.e., a

Maria Chudnovsky: Department of Mathematics, Princeton University, Princeton, NJ 08544, USA

Neil Robertson: Department of Mathematics, Ohio State University, 231 W. 18th Ave., Columbus, Ohio 43210, USA

P. D. Seymour: Department of Mathematics, Princeton University, Princeton, NJ 08544, USA Robin Thomas: School of Mathematics, Georgia Institute of Technology, Atlanta, GA 30332, USA

Mathematics Subject Classification (1991): 05C17 
hole with an odd number of vertices), then $\chi(G)=3>2=\omega(G)$. Similarly, if $G$ is an odd antihole, say on $2 k+1$ vertices, then $\chi(G)=k+1>k=\omega(G)$.

A graph $G$ is perfect if $\chi(H)=\omega(H)$ for every induced subgraph $H$ of $G$; that is, the chromatic number of $H$ is equal to the maximum size of a clique of $H$. Thus odd holes and odd antiholes are not perfect, and neither is any other graph that has an odd hole or an odd hole antihole. Are there any other imperfect graphs? Berge [1] conjectured in 1961 that there are not, and a proof of that is our main result:

\subsection{A graph is perfect if and only if it has no odd hole and no odd antihole.}

In the second half of the paper we will outline our proof. The first part is devoted to discussing the relevance of perfect graphs. This survey is not intended to be exhaustive - we concentrate on the aspects of perfect graphs that we find the most interesting. The subject is much broader than can be covered in our short survey. We refer the reader to the excellent book by Ramírez Alfonsín and Reed [34] for further reading.

\section{Examples of perfect graphs}

A graph $B$ is bipartite if its vertex-set $V(B)$ can be partitioned into two disjoint sets $X, Y$ in such a way that every edge of $B$ has one end in $X$ and the other end in $Y$. In other words, $B$ is bipartite if and only if $\chi(B) \leq 2$. It follows immediately that every bipartite graph is perfect.

Now let $G$ be the complement of a bipartite graph $B$, in symbols $G=\bar{B}$. We claim that $G$ is perfect. Since every induced subgraph of $G$ is the complement of an appropriate subgraph of $B$, it suffices to show that $\chi(G)=\omega(G)$. But $\omega(G)=\alpha(B)$, the maximum size of a stable set of $B$, and $\chi(G)$ is equal to the minimum number of cliques of $B$ covering the vertices of $B$. Thus the statement $\chi(G)=\omega(G)$ is equivalent to the assertion that the vertices of the bipartite graph $B$ can be covered by $\alpha(B)$ edges and vertices. The latter is a classical result of König [23] and can be found in almost every graph theory textbook.

Another two classes of perfect graphs can be obtained from bipartite graphs by means of line graphs. Let $G$ be a graph. The line graph of $G$ is the graph $L(G)$ with vertex-set $E(G)$ in which $e, f \in E(G)$ are adjacent if they share an end in $G$. Now if $B$ is a bipartite graph, then $G=L(B)$ is perfect. To see this, it again suffices to show that $\chi(G)=\omega(G)$. Now $\omega(G)$ is equal to the maximum degree of $B$, and $\chi(G)$ is equal to the edge-chromatic number of $B$, the minimum number of colors needed to color the edges of $B$ in such a way that adjacent edges receive different colors. The latter two numbers are equal in any bipartite graph $B$ by a well-known theorem of König [23].

Similarly, if $B$ is a bipartite graph, then $G=\overline{L(B)}$, the complement of $L(B)$, is perfect. To see this it again suffices to show that $\chi(G)=\omega(G)$. But $\chi(G)$ is the minimum number of cliques of $L(B)$ that cover the vertices of $L(B)$, which in turn is equal to the minimum number of vertices of $B$ that meet all the edges of $B$. On the other hand, $\omega(G)$ is the size of a maximum stable set in $L(B)$, 
which is equal to the maximum size of a matching of $B$. But the maximum size of a matching in a bipartite graph is equal to the minimum number of vertices meeting all the edges of $B$ by another classical theorem of Egerváry [13] and König [24].

Thus we have seen four classes of perfect graphs. The perfection of the first class is easy, and for the other three classes their perfection is equivalent to an old and well-known result in graph theory. By now many more classes have been discovered; by the last count there are 96 known classes of perfect graphs.

\section{The perfect graph theorem}

Notice that in the above examples of perfect or imperfect graphs it was always the case that a graph is perfect if and only if so is its complement. This is not a coincidence, due to the following theorem of Lovász [26], originally conjectured by Berge [1].

3.1 (Perfect Graph Theorem) A graph is perfect if and only if its complement is perfect.

For the proof we will need two lemmas. The first is easy.

3.2 A graph $G$ is perfect if and only if every induced subgraph $H$ of $G$ has a stable set that intersects every maximum clique of $H$.

The second lemma we need is due to Lovász [26].

3.3 (Replication Lemma) Let $G$ be a perfect graph, and let $v \in V(G)$. Define a graph $G^{\prime}$ by adding a new vertex $v^{\prime}$ and joining it to $v$ and all the neighbors of $v$. Then $G^{\prime}$ is perfect.

Proof. By (3.2) it suffices to prove that $G^{\prime}$ itself has a stable set that intersects every maximum clique of $G^{\prime}$. Since $G$ is perfect, it has a coloring using $\omega(G)$ colors; let $S$ be the color class containing $v$. Then $S$ is as desired.

In the proof of (3.1) we will give another characterization of perfect graphs in terms of certain polytopes associated with graphs. Let $G$ be a graph. The stable set polytope of $G$, also known as the vertex packing polytope of $G$, denoted by $\operatorname{STAB}(G)$, is the convex hull in $\mathbf{R}^{V(G)}$ of all incidence vectors of stable sets of $G$. A related polytope is the fractional stable set polytope or fractional vertex packing polytope $\operatorname{QSTAB}(G) \subseteq \mathbf{R}^{V(G)}$ defined by the constraints

$$
\begin{aligned}
x_{v} & \geq 0 \quad \text { for every } v \in V(G), \\
\sum_{v \in V(K)} x_{v} & \leq 1 \quad \text { for every clique } K \text { in } G .
\end{aligned}
$$

Since the incidence vector of every stable set satisfies (1) and (2), we have $\operatorname{STAB}(G) \subseteq \operatorname{QSTAB}(G)$. The following theorem, which also incorporates results of Chvátal [2] and Fulkerson [14,15], implies the Perfect Graph Theorem. Our treatment follows [28]. 
3.4 For any graph $G$, the following conditions are equivalent.

(i) $G$ is perfect,

(ii) $\operatorname{STAB}(G)=Q S T A B(G)$,

(iii) $\bar{G}$ is perfect,

(iv) $\operatorname{STAB}(\bar{G})=Q S T A B(\bar{G})$.

Proof. (i) $\Rightarrow$ (ii). Let $x$ satisfy (1) and (2). By rational approximation we may assume that $x$ is rational. Take an integer $N$ such that $y=N x$ is an integer vector. By (1), $y_{v} \geq 0$ for all $v \in V(G)$. Let $Y_{v}(v \in V(G))$ be disjoint sets with $\left|Y_{v}\right|=y_{v}$, and let $\bar{H}$ be the graph with vertex-set $\bigcup_{v \in V(G)} Y_{v}$ in which a vertex of $Y_{v}$ is adjacent to a vertex of $Y_{u}$ if and only if either $v=u$ or $v$ is adjacent to $u$ in $G$. By a repeated application of the Replication Lemma (3.3) the graph $H$ is perfect. By $(2)$ we have $\omega(H) \leq N$, and hence $V(H)$ can be covered by $N$ disjoint stable sets of $H$. Let $q_{1}, q_{2}, \ldots, q_{N}$ be the incidence vectors of the corresponding stable sets of $G$. Then $x=\frac{1}{N}\left(q_{1}+q_{2}+\cdots+q_{N}\right)$, as desired.

(ii) $\Rightarrow$ (iii) Since condition (ii) is inherited by induced subgraphs of $G$, by (3.2) it suffices to prove that $G$ has a clique that intersects every maximum stable set of $G$. We prove this assertion by induction on $|V(G)|$. We may assume that every vertex belongs to a maximum stable set of $G$, for otherwise we may delete such vertex and proceed by induction. Let $X$ be the subset of $\operatorname{STAB}(G)$ consisting of all vectors $x$ with $\sum_{v \in V(G)} x_{v}=\alpha(G)$. Then $X$ is a face of $\operatorname{QSTAB}(G)$, and hence one of the inequalities (1), (2) is satisfied with equality for every vector in $X$. Since every vertex of $G$ belongs to a maximum stable set of $G$, it follows that none of the inequalities (1) is satisfied with equality for all $x \in X$, and hence one of the inequalities (2) is. Thus there exists a clique of $G$ which meets every maximum stable set of $G$, as desired.

The remaining two implications follow by applying the previous arguments to the complement of $G$.

\section{Integrality of polyhedra}

Let $A$ be a 0,1 -matrix and consider the following linear program:

$$
\max c \cdot x \text { subject to } x \geq 0 \text { and } A x \leq 1 \text {. }
$$

For which matrices $A$ is it true that for every objective function $c$, the linear program has integral optimum solution? This is an important question, because solving integer programs is an NP-hard problem, whereas efficient algorithms exist to solve linear programs. It turns out that the answer to our question leads directly to perfect graphs.

We say that the $i$ th row of a matrix $A=\left(a_{i j}\right)$ is undominated if there is no row index $j \neq i$ such that $a_{i l} \leq a_{j l}$ for all $l$. Let $G$ be a graph with $V(G)=\left\{v_{1}, v_{2}, \ldots, v_{n}\right\}$, and let $K_{1}, K_{2}, \ldots, K_{m}$ be its (inclusion-wise) maximal cliques. We define the maximal clique versus vertex incidence matrix of $G$ to be the $m \times n$ matrix $A=\left(a_{i j}\right)$, where $a_{i j}=1$ if $v_{j} \in K_{i}$, and $a_{i j}=0$ otherwise. The following is a result of Chvátal [2]. 
4.1 The linear program (3) has an integral optimum solution for every objective function $c$ if and only if the undominated rows of $A$ form the maximal clique versus vertex incidence matrix of a perfect graph.

Proof. To prove "only if" let $A=\left(a_{i j}\right)$ be an $m \times n$ matrix, and assume that (3) has an integral optimum for every nonnegative objective function $c$. We may assume that $A$ has no dominated rows (by deleting them). Let $G$ be the graph with vertex-set $\{1,2, \ldots, n\}$ in which $i$ is adjacent to $j$ if and only if $a_{l i}=a_{l j}=1$ for some row index $l$. We first show that $A$ is a maximal clique versus vertex incidence matrix of $G$. Indeed, if that were not the case, then $G$ has a clique $K$ such that no row of $A$ has ones in the positions corresponding to the vertices of $K$ and possibly elsewhere. Choose such $K$ with $|K|$ minimal. Then clearly $|K| \geq 3$ by construction. By the minimality of $K$, for every $v \in K$ there exists a row with ones in columns corresponding to $K-\{v\}$. Let $c$ be the incidence vector of $K$. Then $c \cdot x \leq 1$ for every integral vector $x \in \mathbf{R}^{V(G)}$ satisfying $x \geq 0$ and $A x \leq 1$, for such $x$ must be the incidence vector of a stable set. Now let $y \in \mathbf{R}^{V(G)}$ be the vector whose coordinates are $\frac{1}{|K|-1}$ in positions corresponding to elements of $K$, and all other coordinates are 0 . Then $A y \leq 1$, and yet $c \cdot y=\frac{|K|}{|K|-1}>1$, a contradiction. This proves that $A$ is a maximal clique versus vertex incidence matrix of $G$, and hence the polyhedron $\left\{x \in \mathbf{R}^{V(G)}: x \geq 0\right.$ and $\left.A x \leq 1\right\}$ is precisely $\operatorname{QSTAB}(G)$. Now the integrality of optima implies that every vertex of $\operatorname{QSTAB}(G)$ is integral, and hence is a characteristic vector of a stable set. Thus $\operatorname{STAB}(G)=\operatorname{QSTAB}(G)$, and hence $G$ is perfect by $(3.4)$.

To prove the "if" part, let $G$ be perfect. We may assume that $A$ itself is the maximal clique versus vertex incidence matrix of $G$. By (3.4) the linear program (3) is equivalent to maximizing $c \cdot x$ subject to $x \in \operatorname{STAB}(G)$, which clearly attains its optimum at a characteristic vector of a stable set, and hence has an integral optimum solution, as desired.

\section{Berge's motivation: Shannon capacity}

Let us go back in history and review what motivated Berge to introduce perfect graphs. Let us consider the transmission of symbols from some finite input alphabet $\Sigma$ through a discrete memoryless channel. Certain symbols may be confused during transmission, and we are interested in how many $n$-symbol error-free messages there are.

To be more precise, with every $a \in \Sigma$ we associate symbols $a_{1}, a_{2}, \ldots$ of some output alphabet. The meaning of this is that when $a$ is sent through the channel, one of $a_{1}, a_{2}, \ldots$ is received, say according to some probability distribution. However, in this model we are interested in transmissions that are $100 \%$ errorfree, and so we define confoundability as follows. Let $a \in \Sigma$ be as above, and let $b \in \Sigma$ have $b_{1}, b_{2}, \ldots$ as the corresponding members of the output alphabet. We say that $a$ and $b$ are confoundable if $a_{i}=b_{j}$ for some $i, j$, and otherwise we say that they are unconfoundable. Finally, we say that two words $x, y \in \Sigma^{t}$ of 
length $t$ are unconfoundable if for at least one coordinate $i$ the corresponding entries $x_{i}$ and $y_{i}$ are unconfoundable.

As an example, let $\Sigma=\{a, b, c, d, e\}$, where the confoundable pairs are precisely $a b, b c, c d$, de, ea. In that case the symbols $a, c$ may be sent without danger of confusion, and hence there are at least $2^{n} n$-symbol error-free messages obtained by taking all $n$-element sequences of the symbols $a$ and $c$. But we can do better. The 2-symbol messages $a b, b d, c a, d c, e e$ are pairwise unconfoundable (for instance $a$ may be confused with $b$, but $b$ cannot be confused with $d$, and hence the pairs $a b$ and $b d$ are unconfoundable), and by forming arbitrary words composed of those two-letter words we see that there are at least $5^{\lfloor n / 2\rfloor} n$-symbol error-free messages, an improvement over the earlier bound. An asymptotic study of the bit-per-symbol error-free transmission rate leads to the notion of Shannon capacity of a graph [39], as follows.

Let $G$ be the graph with vertex-set $\Sigma$ and edge-set all pairs of unconfoundable elements of $\Sigma$. Thus we are interested in maximum cliques of $G^{t}$, where $G^{t}$ is the graph with vertex-set all $t$-tuples of vertices of $G$ in which two such $t$-tuples are adjacent if and only if for some coordinate the corresponding entries are adjacent in $G$. The Shannon capacity of $G$ is defined as

$$
\lim _{n \rightarrow \infty} \frac{1}{n} \log \omega\left(G^{n}\right) .
$$

It is easy to see that the limit exists. Moreover, we have the following inequalities: $\omega^{n}(G) \leq \omega\left(G^{n}\right) \leq \chi\left(G^{n}\right) \leq \chi^{n}(G)$. Thus it follows that if $\chi(G)=\omega(G)$, then the Shannon capacity is equal to the logarithm of this value. This raises the question of what are the minimal graphs that do not satisfy $\chi(G)=\omega(G)$, and that lead Berge to formulate his influential conjectures.

The Shannon capacity is a notoriously difficult parameter to compute. For instance, a celebrated result of Lovász [27] says that the Shannon capacity of the cycle of length five is $\frac{1}{2} \log 5$. The lower bound follows from our discussion two paragraphs above, but the upper bound is a deep result. Lovász' proof uses a new parameter, based on geometric representation of graphs, the so-called theta function of graphs. The theta function played a crucial role in the theory of Grötschel, Lovász and Schrijver that we discuss in the next section.

Incidentally, the Shannon capacity is not known for many small graphs. For instance, it is not known for odd cycles of length at least seven.

\section{Miscellaneous connections}

Grötschel, Lovász and Schrijver [19] developed a general theory of geometric optimization based on the ellipsoid method [22]. One consequence of this theory is that an optimal coloring and a maximum clique of a perfect graph can be found in polynomial time. More generaly, the theory provided the first theoretically efficient algorithm for semi-definite programming. Semi-definite programs are linear programs over the cones of semi-definite matrices. Practitioners have been formulating and solving semi-definite programs for decades, although they knew 
of no theoretically efficient algorithm to solve them. As pointed out in [35], algorithms to solve semi-definite programs grew out of the theory of perfect graphs.

An application of perfect graphs to municipal services is described in [41] and [42]. A more recent application area of perfect graphs has been investigated by Gerke, McDiarmid and Reed [16-18,31,32]. Motivated by the radio channel assignment problem they introduced and studied a new parameter, called the imperfection ratio. There are several equivalent definitions, but the easiest for us to state is the following: the imperfection ratio of a graph, denoted by $\operatorname{imp}(G)$, is the minimum number $t$ such that $\operatorname{QSTAB}(G) \subseteq t \operatorname{STAB}(G)$. Thus by (3.4) $\operatorname{imp}(G)=1$ if and only if $G$ is perfect. Furthermore, $\operatorname{imp}(G)=\operatorname{imp}(\bar{G})$, generalizing (3.1). We refer to [31] for more details, including background on the channel assignment problem.

\section{Graph entropy}

Körner [25] studied the following problem. Let $G$ be a graph, and let $P=\left(p_{v}\right.$ : $v \in V(G)$ ) be a probability distribution on $V(G)$. We will think of $V(G)$ as a finite alphabet whose elements are being emitted by a discrete memoryless and stationary information source according to the probability distribution $P$. Adjacency in $G$ is interpreted as distinguishability. Two $t$-tuples of vertices of $G$ are distinguishable if they are distinguishable in at least one coordinate, and are indistinguishable otherwise.

We wish to examine the performance of a best possible encoding. Here for $\epsilon>0$ an $\epsilon$-encoding is a mapping from $V(G)^{t} \rightarrow M$, where $M$ is some set, such that there is a set $\Omega \subset V(G)^{t}$ of probability (in the product space) at least $1-\epsilon$ such that every two distinguishable $t$-tuples from $\Omega$ are mapped onto different elements of $M$. The rate of this encoding is defined as $\frac{\log |M|}{t}$. Let $R(G, P, t, \epsilon)$ denote the smallest possible rate over all possible $\epsilon$-encodings. Of interest is the value $\liminf _{\epsilon \rightarrow 0} \liminf _{t \rightarrow \infty} R(G, P, t, \epsilon)$. This is the graph entropy $H(G, P)$, introduced by Körner [25]. Körner gave several descriptions of $H(G, P)$, including the following.

7.1 Let $G$ be a graph, and let $P$ be a probability distribution on $V(G)$. Then $H(G, P)=\min _{x \in \operatorname{STAB}(G)} \sum_{v \in V(G)} p_{v} \log \frac{1}{p_{v}}$.

If $G$ is the complete graph, then $H(G, P)$ specializes to the classical notion of entropy of a probability distribution, namely $H(P)=\sum_{i=1}^{n} p_{i} \log \frac{1}{p_{i}}$. From an information theory point of view it is interesting to study graphs for which $H(G, P)+H(\bar{G}, P)=H(P)$ for every probability distribution $P$. By a theorem of Cziszár, Körner, Lovász, Marton and Simonyi [12] this holds if and only if $G$ is perfect. Simonyi [40] recently generalized this by showing that $\max _{P}(H(G, P)+H(\bar{G}, P)-H(P))=\log \operatorname{imp}(G)$, where $\operatorname{imp}(G)$ is the imperfection ratio mentioned earlier.

Finally, let us mention that graph entropy and perfection played important roles in the sorting algorithm of Kahn and Kim [21]. 


\section{Outline of the proof}

We now come to the second part of the paper. In the remainder of the text we will outline our proof of (1.1). It is customary to call a graph Berge if it has no odd hole and no odd antihole. Thus in order to prove (1.1) we must show that every Berge graph is perfect. We use a strategy originated by Conforti, Cornuéjols and Vušković. They conjectured that every Berge graph either belongs to one of the four classes introduced in Section 2, or has a separation of one of two kinds. This is a reasonable plan, because similar approach was successful for many other graph theory problems, for instance $[29,30,36,37,43]$, and many others. We were able to prove this conjecture, but to deduce (1.1) we needed to prove a minor modification of it. Let us first introduce the two kinds of separation. A 2-join in $G$ is a partition $\left(X_{1}, X_{2}\right)$ of $V(G)$ so that there exist disjoint nonempty sets $A_{i}, B_{i} \subseteq X_{i}(i=1,2)$ satisfying:

- every vertex of $A_{1}$ is adjacent to every vertex of $A_{2}$, every vertex of $B_{1}$ is adjacent to every vertex of $B_{2}$, and there are no other edges between $X_{1}$ and $X_{2}$

- for $i=1,2$, every component of $G \mid X_{i}$ meets both $A_{i}$ and $B_{i}$, and

- for $i=1,2$, if $\left|A_{i}\right|=\left|B_{i}\right|=1$ and $G \mid X_{i}$ is a path joining the members of $A_{i}$ and $B_{i}$, then it has odd length $\geq 3$.

Cornuéjols and Cunningham [11] (see also [10]) proved that no minimal counterexample to (1.1) has a 2-join. More precisely, they proved:

\subsection{No minimally imperfect graph has a 2-join.}

Moreover, 2-joins afford a decomposition into two smaller graphs such that the original graph is Berge if and only if both the new graphs are Berge, and the same holds for perfection. By (3.1) it follows that the complement of a minimal counterexample to (1.1) also has no 2-join.

Let us discuss skew partitions now. A set $X \subseteq V(G)$ is connected if $G \mid X$ is connected or if $X$ is empty; and anticonnected if $\bar{G} \mid X$ is connected. A skew partition in $G$ is a partition $(A, B)$ of $V(G)$ so that $A$ is not connected and $B$ is not anticonnected. Chvátal [3] conjectured that no minimal imperfect graph has a skew partition. This follows from (1.1): by (1.1) the only minimal imperfect graphs are odd holes and odd antiholes, and it is easy to check that those graphs have no skew partition. But in order to be able to use Chvátal's conjecture we would need a proof from first principles, and we were unable to find one. However, it turned out that for our purposes a restricted class of skew partitions sufficed, what we call even skew partitions.

An antipath is a subgraph whose complement is a path. The length of a path is the number of edges in it and the length of an antipath is the number of edges in its complement. A skew partition $(A, B)$ is even if every induced path of length at least two with ends in $B$ and with interior in $A$ is even, and every induced antipath of length at least two with ends in $A$ and with interior in $B$ is even. The significance of even skew partitions is that we can prove a variant of Chvátal's skew partition conjecture for them. Let us say a minimum imperfect 
graph is a Berge graph $G$ such that $G$ is not perfect, but every Berge graph $H$ with $|V(H)|<|V(G)|$ is perfect.

8.2 No minimum imperfect graph has an even skew partition.

We give a proof of (8.2) at the end of this section. Our main result is a version of the conjecture of Conforti, Cornuéjols and Vušković, with skew partitions replaced by even skew partitions. It turns out that if we do that, then we need another basic class, the following.

Let $H$ be a bipartite graph, with bipartition $(A, B)$. For each vertex $v \in V(H)$ take two new vertices $s_{v}, t_{v}$, and make a graph $G$ with $V(G)=\left\{s_{v}, t_{v}: v \in\right.$ $V(H)\}$. The edges of $G$ are as follows:

- for $v \in V(H), s_{v}$ is adjacent to $t_{v}$ if $v \in A$, and $s_{v}$ is nonadjacent to $t_{v}$ if $v \in B$.

- for distinct $u, v \in A$, there are no edges between $s_{u}, t_{u}$ and $s_{v}, t_{v}$

- for distinct $u, v \in B$, both $s_{u}, t_{u}$ are adjacent to both $s_{v}, t_{v}$.

- for $u \in A$ and $v \in B$, there are exactly two edges joining one of $s_{u}, t_{u}$ to one of $s_{v}, t_{v}$; if $u v \in E(H)$ then $s_{u} s_{v}$ and $t_{u} t_{v}$ are edges of $G$, and otherwise $s_{u} t_{v}$ and $t_{u} s_{v}$ are edges of $G$.

We call such a graph $G$ a double split graph. Let us say a graph $G$ is basic if either $G$ or $\bar{G}$ is bipartite or is the line graph of a bipartite graph, or is a double split graph. (Note that if $G$ is a double split graph then so is $\bar{G}$.) It is easy to see that all double split graphs are perfect, and thus all basic graphs are perfect by the results discussed in Section 2 .

If $X, Y \subseteq V(G)$ are disjoint, we say $X$ is complete to $Y$ (or the pair $(X, Y)$ is complete) if every vertex in $X$ is adjacent to every vertex in $Y$; and we say $X$ is anticomplete to $Y$ if there are no edges between $X$ and $Y$. An $M$-join in $G$ is a partition of $V(G)$ into six nonempty sets, $(A, B, C, D, E, F)$, so that:

- every vertex in $A$ has a neighbor in $B$ and a nonneighbor in $B$, and vice versa

- the pairs $(C, A),(A, F),(F, B),(B, D)$ are complete, and

- the pairs $(D, A),(A, E),(E, B),(B, C)$ are anticomplete.

M-joins are closely related to "homogenous sets" of Chvátal and Sbihi [4], and it follows from their work that no minimally imperfect graph has an M-join. Our main result is as follows.

8.3 For every Berge graph $G$, either $G$ is basic, or one of $G, \bar{G}$ admits a 2-join, or $G$ admits an $M$-join, or $G$ admits an even skew partition.

The introduction of double split graphs is necessary, because, in general, even though double split graphs have skew partitions, they do not have even ones. The first author has shown in her $\mathrm{PhD}$ thesis [5] that $\mathrm{M}$-joins are not needed in the sense that the above theorem remains true if we omit the outcome that $G$ has an M-join. However, to prove this strengthening required an amount of effort comparable to the proof of (8.3) itself. By (8.1), (8.2) and the preceding discussion, theorem (8.3) implies (1.1). 
In the remainder of this section we prove (8.2). In fact, we prove something seemingly stronger. Let us say a skew partition $(A, B)$ in a graph $G$ is rigid if there exists a maximal anticonnected set $B_{1} \subseteq B$ such that the graph obtained from $G$ by adding a new vertex joined precisely to all the vertices in $B_{1}$ is Berge. It follows that $(A, B)$ is rigid if and only if every induced path of length at least two with both ends in $B_{1}$ and interior in $A$ is even, and every induced antipath of length at least two with both ends in $A$ and interior in $B_{1}$ is even. Thus every even skew partition is rigid, and hence (8.2) is implied by the following. Our proof incorporates results of Chvátal [3] and Hoàng [20].

\subsection{No minimum imperfect graph has a rigid skew partition.}

Proof. Suppose for a contradiction that $G$ is a minimum imperfect graph with a rigid skew partition $(A, B)$, and let $B_{1}$ be as in the definition of rigid. If one component of $A$ has only one vertex, then by considering the vertex-set of that component we find that $(B, A)$ is a rigid skew partition of $\bar{G}$. Thus we may assume that if some component of $A$ has only one vertex, then $\left|B_{1}\right|=1$.

Let $A_{1}$ be the vertex-set of a component of $G \mid A$, and let $A_{2}=A-A_{1}$. For $i=1,2$ let $G_{i}$ be the subgraph of $G$ induced by $A_{i} \cup B$. Let $k=\omega(G)$ and $s=\omega\left(B_{1}\right)$. (For $Z \subseteq V(G)$ we define $\omega(Z)$ to be the size of a largest clique contained in $Z$.) We need the following claim.

Claim. For $i=1,2$ there exists a set $X_{i} \subseteq V\left(G_{i}\right)$ such that $X_{i} \cap B=B_{1}$, $\omega\left(X_{i}\right)=s$ and $\omega\left(G_{i} \backslash X_{i}\right) \leq k-s$.

It suffices to prove the claim for $i=1$. Assume first that $\left|B_{1}\right|=1$. Since $G_{1}$ is perfect by the minimality of $G$, it has a coloring using $k$ colors. Let $X_{1}$ be the color class containing the unique element of $B_{1}$. Then $X_{1}$ is as desired, because $B_{1}$ is complete to $B-B_{1}$. Thus we may assume that $\left|B_{1}\right|>1$, and hence by the choice of $(A, B)$ above we have $\left|A_{1}\right| \geq 2$ and $\left|A_{2}\right| \geq 2$.

Let $G_{1}^{\prime}$ be the graph obtained from $G_{1}$ by adding a vertex $z$ joined to precisely the vertices of $B_{1}$. Then $G_{1}^{\prime}$ is Berge by the rigidity of $(A, B)$, and hence is perfect by the minimality of $G$ and the fact that $\left|A_{2}\right| \geq 2$. Let $G_{1}^{\prime \prime}$ be obtained from $G_{1}^{\prime}$ by replacing $z$ by a clique $K$ of size $k-s$, all of whose vertices are adjacent to $B_{1}$ and to no other vertex of $G_{1}$. Then $G_{1}^{\prime \prime}$ is perfect by $(3.3)$. We have $\omega\left(G_{1}^{\prime \prime}\right) \leq k$ by construction, and hence $G_{1}^{\prime \prime}$ has a coloring using $k$ colors. Exactly $k-s$ of those colors appear on $K$, and hence exactly $s$ colors appear on vertices of $B_{1}$. Let $X_{1}$ be the set of all vertices of $G_{1}$ colored using one of those $s$ colors. Since $B_{1}$ is complete to $B-B_{1}$, it follows that $X_{1}$ is as desired. This proves the claim.

Now let $X_{1}$ and $X_{2}$ be as in the claim. It follows that $\omega\left(X_{1} \cup X_{2}\right)=s$ and $\omega\left(G \backslash\left(X_{1} \cup X_{2}\right)\right) \leq k-s$. Thus $G \mid\left(X_{1} \cup X_{2}\right)$ can be colored using $s$ colors, and $G \backslash\left(X_{1} \cup X_{2}\right)$ can be colored using $k-s$ colors, because both those graphs are perfect by the minimality of $G$. It follows that $G$ is $k$-colorable, contrary to the fact that $G$ is minimum imperfect. 


\section{Line graphs}

In the rest of the paper we outline the proof of (8.3). The first step is to show that if $G$ contains a line graph of a bipartite subdivision of a "large" 3-connected graph, then either $G$ is itself a line graph of a bipartite graph, or it has one of the separations mentioned in (8.3).

9.1 Let $G$ be a Berge graph, let $J$ be a 3-connected graph, let $H$ be a bipartite subdivision of $J$, and let $L(H)$ be an induced subgraph of $G$. Assume further that $H \neq K_{3,3}$, and that if $J=K_{4}$, then some edge of every 4-cycle of $J$ is subdivided in $H$. Then $G$ is a line graph of a bipartite graph, or has a 2-join or has an even skew partition.

Let us sketch the main steps in the proof of (9.1). First, it is convenient to enlarge $L(H)$ into a certain auxiliary structure, which we call a $J$-strip system. Let $J$ be 3 -connected, and let $G$ be Berge. A $J$-strip system in $G$ is a pair $(S, N)$, where $S_{u v}=S_{v u} \subseteq V(G)$ for each edge $u v$ of $J$, and $N_{v} \subseteq V(G)$ for each vertex $v$ of $J$ such that (for $u v \in E(J)$, a $u v$-rung means an induced path $R$ of $G$ with ends $s, t$ say, where $V(R) \subseteq S_{u v}$, and $s$ is the unique vertex of $R$ in $N_{u}$, and $t$ is the unique vertex of $R$ in $N_{v}$ )

(i) the sets $S_{u v}(u v \in E(J))$ are pairwise disjoint,

(ii) for each $u \in V(J), N_{u} \subseteq \bigcup\left(S_{u v}: v \in V(J)\right.$ adjacent to $\left.u\right)$,

(iii) for each $u v \in E(J)$, every vertex of $S_{u v}$ is in a $u v$-rung,

(iv) if $u v, w x \in E(J)$ with $u, v, w, x$ all distinct, then there are no edges between $S_{u v}$ and $S_{w x}$

(v) if $u v, u w \in E(J)$ with $v \neq w$, then $N_{u} \cap S_{u v}$ is complete to $N_{u} \cap S_{u w}$, and there are no other edges between $S_{u v}$ and $S_{u w}$.

Now $L(H)$ gives rise to a $J$-strip system $(S, N)$ as follows. First notice that $V(J) \subseteq V(H)$, and that each edge $u v \in E(J)$ corresponds to a path in $H$. Let $f_{1}, f_{2}, \ldots, f_{k}$ be the edges of that path, in order, such that $f_{1}$ is incident with $u$. Then $f_{1}, f_{2}, \ldots, f_{k}$ is the vertex-set of an induced path of $L(H)$. We put $f_{1}$ into $N_{u}, f_{k}$ into $N_{v}$, and define $S_{u v}:=\left\{f_{1}, f_{2}, \ldots, f_{k}\right\}$. By repeating this for every edge of $J$ we arrive at a $J$-strip system, which we call the $J$-strip system derived from $L(H)$.

If $(S, N)$ is a $J$-strip system in $G$, we define $V(S, N)$ to be the union of $S_{u v}$ over all edges of $J$. Thus $N_{v} \subseteq V(S, N)$ for every $v \in V(J)$. We say that a set $X$ saturates the $J$-strip system $(S, N)$ if for every $u \in V(J)$ there is at most one neighbor $v$ of $u$ in $J$ such that $N_{u} \cap S_{u v} \nsubseteq X$. A vertex $u \in V(G)-V(S, N)$ is major if its neighbors in $V(S, N)$ saturate $(S, N)$, and minor otherwise. A set $X \subseteq V(S, N)$ is local if either $X \subseteq N_{v}$ for some $v \in V(J)$, or $X \subseteq S_{u v}$ for some edge $u v \in E(J)$. For the proof of (9.1) we first choose $J$ with $|V(J)|$ maximum, start with the $J$-strip system derived from $L(H)$, and enlarge it to a $J$-strip system with $V(S, N)$ maximum. The advantage of that is that now we have the following lemma:

9.2 Let $F \subseteq V(G)-V(S, N)$ be connected and contain no major vertices. Then the set of neighbors in $V(S, N)$ of vertices in $F$ is a local set. 

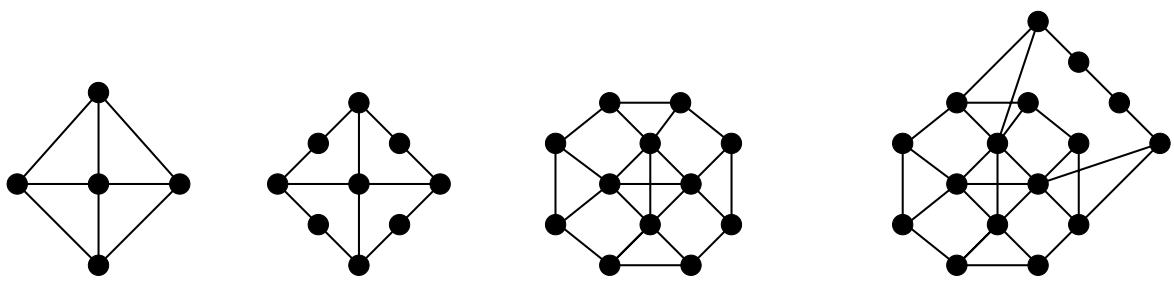

Fig. 1. $J, H, L(H)$ and $G$

We will not give a proof of this lemma, but let us point to Figure 1 for illustration. Let $(S, N)$ be the $J$-strip system derived from $L(H)$, where $J$ and $H$ are as in Figure 1. There is a unique component $F$ of $V(G)-V(S, N)$, and the neighbors of $F$ do not form a local set. But the strip system is not maximal: the set $F$ can be added to the $J$-strip system $(S, N)$.

Now for the proof of (9.1). Suppose first that there is a major vertex, and let $Y$ be a maximal anticonnected set of major vertices. Let $X$ be the set of common neighbors of vertices in $Y$. It can be shown that $X$ saturates the strip system, and from there it is not hard to produce first a skew partition, and then an even skew partition.

Thus we may assume that there are no major vertices. If there is a component $F$ of $G \backslash V(S, N)$ whose set of neighbors is a subset of $N_{v}$ for some $v \in V(J)$, then $\left(V(G)-N_{v}, N_{v}\right)$ is a skew partition, and it is not hard to convert it into an even skew partition. On the other hand we may assume that $G \neq L(H)$, and so by (9.2) there is an edge $u v \in E(J)$ such that either $S_{u v}$ is not a path, or some component of $G \backslash V(S, N)$ has all its neighbors in $S_{u v}$. Let $F$ be the union of all such components. Then the partition $\left(F \cup S_{u v}, V(G)-F-S_{u v}\right)$ is a 2 -join in $G$. This completes the sketch of the proof of $(9.1)$.

If $H=K_{3,3}$, then the same argument works, except for the step where we deduce that the set of common neighbors of a maximal anticonnected component of major vertices saturates the strip system. However, if that outcome does not hold, then we find that there is a bipartite subdivision $H^{\prime}$ of $K_{3,3}$, itself not equal to $K_{3,3}$, such that $L\left(H^{\prime}\right)$ is an induced subgraph of the complement of $G$. In that case we apply $(9.1)$ to $H^{\prime}$ and $\bar{G}$. This proves

9.3 Let $G$ be a Berge graph, let $J$ be a 3-connected graph, let $H$ be a bipartite subdivision of $J$, and let $L(H)$ be an induced subgraph of $G$. Assume further that if $J=K_{4}$, then some edge of every 4 -cycle of $J$ is subdivided in $H$. Then $G$ or its complement is a line graph of a bipartite graph, or has a 2-join or has an even skew partition.

Let us now assume that $J=K_{4}$, and that no edge of some 4-cycle of $J$ is subdivided in $H$. Then $L(H)$ is pictured in Figure 2 ; let $P_{1}, P_{2}$ and $v_{1}, v_{2}, v_{3}, v_{4}$ be as in that figure. Let $Q_{1}$ denote the antipath with vertex-set $\left\{v_{1}, v_{3}\right\}$, and let $Q_{2}$ denote the antipath with vertex-set $\left\{v_{2}, v_{4}\right\}$. Notice that if one of $P_{1}$, $P_{2}$ has length at least two (and hence at least three, because they are odd), then $L(H)$ has a 2-join, whereas if they both have length one, then $L(H)$ is a 


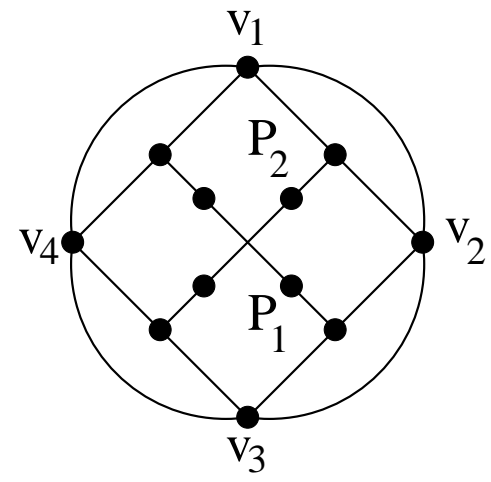

Fig. 2. Line graph of a subdivision of $K_{4}$

double split graph. Thus our strategy is as follows. First, we collect all such paths $P_{1}, P_{2}, \ldots$ and antipaths $Q_{1}, Q_{2}, \ldots$ More generally, we enlarge them into strips and "antistrips", and maximize the resulting structure $S$. We divide vertices of $V(G)-V(S)$ into major and minor similarly as before. Here the situation is nicer, because the structure is invariant under taking complements, and taking complements turns minor vertices into major ones, and vice versa. As before, if there is a major vertex, then a maximal anticonnected component of such vertices gives rise to a skew partition, and similarly for connected components of minor vertices. Finally, if all of $G$ falls into the structure, then either the structure proves that $G$ is a double split graph, or $G$ has a 2 -join. Thus we have

9.4 Let $G$ be a Berge graph, let $H$ be a bipartite subdivision of a 3-connected graph, and let $L(H)$ be an induced subgraph of $G$. Then $G$ is a double split graph, or has an even skew partition, or it or its complement is a line graph of a bipartite graph, or has a 2-join.

\section{Prisms}

A triangle in a graph $G$ is a cycle of length three. Let $a_{1} a_{2} a_{3}$ and $b_{1} b_{2} b_{3}$ be disjoint triangles, and let $P_{1}, P_{2}, P_{3}$ be three vertex-disjoint induced paths in $G$, where $P_{i}$ has ends $a_{i}$ and $b_{i}$, and at least one of $P_{1}, P_{2}, P_{3}$ has length at least two. Assume further that for $1 \leq i<j \leq 3$ there are no edges between $P_{i}$ and $P_{j}$, except for $a_{i} a_{j}$ and $b_{i} b_{j}$. In those circumstances we say that $P_{1} \cup P_{2} \cup P_{3}$ is a prism in $G$. Thus a prism is the line graph of the graph consisting of three internally disjoint paths joining the same pair of vertices. A version of the following result was suggested to the third author by Kristina Vušković.

10.1 Let $G$ be a Berge graph containing a prism such that neither $G$ nor $\bar{G}$ has an induced subgraph isomorphic to the line graph of a bipartite subdivision of a 3-connected graph. Then either one of $G, \bar{G}$ admits a 2-join, or $G$ admits an even skew partition, or $G$ admits an M-join. 
If $G$ is Berge, and $P_{1}, P_{2}, P_{3}$ are the paths of a prism in $G$, then it is easy to see that $P_{1}, P_{2}, P_{3}$ have the same parity. If they are all even, then (10.1) can be proven by a method analogous to the proof of (9.1). However, the proof is much harder when $P_{1}, P_{2}, P_{3}$ are odd. We refer to [6] for the details.

There is another graph that can be excluded by similar methods. A double diamond is the graph with vertex-set $\left\{a_{1}, a_{2}, a_{3}, a_{4}, b_{1}, b_{2}, b_{3}, b_{4}\right\}$ and the following adjacencies: every two $a_{i}$ 's are adjacent except for $a_{3} a_{4}$, every two $b_{i}$ 's are adjacent except for $b_{3} b_{4}$, and $a_{i}$ is adjacent to $b_{i}$ for all $i=1,2,3,4$.

10.2 Let $G$ be a Berge graph such that no induced subgraph of $G$ or $\bar{G}$ is isomorphic to a prism or the line graph of a bipartite subdivision of a 3-connected graph. If the double diamond is an induced subgraph of $G$, then either $G$ or $\bar{G}$ admits a 2-join, or $G$ admits an even skew partition.

\section{Skew partitions revisited}

Let us say a Berge graph $G$ is bipartisan if no induced subgraph of $G$ or $\bar{G}$ is isomorphic to the double diamond, a prism, or the line graph of a bipartite subdivision of a 3-connected graph. In view of (9.4), (10.1) and (10.2) it suffices to prove (8.3) for bipartisan graphs. From (8.1), (8.2) and the perfection of basic graphs we deduce that every minimum imperfect graph is bipartisan.

It turns out that stronger theorems hold for bipartisan graphs, and in this section we discuss one such result. It can be shown that if a bipartisan graph has a skew partition, then it has an even skew partition. Thus in the remainder of the proof of (8.3) we do not need to concern ourselves with checking whether a particular skew partition is even. Here we confine ourselves to proving the essential part of Chvátal's skew partition conjecture:

\subsection{No minimum imperfect graph admits a skew partition.}

In the proof we will need the following beautiful and powerful theorem of Roussel and Rubio [38]. (We proved it independently, in joint work with Carsten Thomassen, but Roussel and Rubio found it earlier.) We use it many times in our proof of (1.1). So far we have not shown enough detail to demonstrate its usefulness, but its time has come.

11.2 Let $G$ be a Berge graph, let $X \subseteq V(G)$ be anticonnected, and let $P$ be an induced path in $G \backslash X$ of odd length, such that both ends of $P$ are complete to $X$. Then either

(i) some internal vertex of $P$ is complete to $X$, or

(ii) the path $P$ has length at least five and there exists an induced path $Q$ with both ends in $X$ such that $P$ and $Q$ has the same interior, or

(iii) the path $P$ has length three and there is an induced odd antipath joining the internal vertices of $P$ with interior in $X$.

Please note that if (ii) above holds, then $G$ has a prism. Thus for bipartisan graphs we have the following simpler version. 
11.3 Let $G$ be a bipartisan Berge graph, let $X \subseteq V(G)$ be anticonnected, and let $P$ be an induced path in $G \backslash X$ of odd length, such that both ends and no internal vertex of $P$ are complete to $X$. Then the path $P$ has length three and there is an induced odd antipath joining the internal vertices of $P$ with interior in $X$.

Since, as noted above, every minimum imperfect graph is bipartisan, (11.1) follows from (8.4) and the following result.

11.4 If a bipartisan Berge graph has a skew partition, then it has a rigid skew partition.

Proof. Let $G$ be a bipartisan Berge graph, and let $(A, B)$ be a skew partition in $G$ chosen so that $B$ is minimal with respect to inclusion. Assume first that some $v \in B$ has no neighbor in some component of $G \mid A$. Since $(A \cup\{v\}, B-\{v\})$ is not a skew partition of $G$ by the minimality of $B$, we deduce that $B-\{v\}$ is anticonnected. Thus $v$ is adjacent to every vertex of $B-\{v\}$, and hence by considering the anticonnected component $\{v\}$ of $G \mid B$ we deduce that $(A, B)$ is rigid, as desired. Thus we may assume that every $v \in B$ has a neighbor in every component of $G \mid A$.

We now prove the following claim.

Claim. Let $B_{1}$ be a maximal anticonnected subset of $B$. If some vertex $u \in A$ is adjacent to every vertex of $B_{1}$, then $(A, B)$ is rigid.

To prove the claim let first $P$ be an induced path of length at least two with both ends in $B_{1}$ and interior in $A$. We must prove that $P$ is even. The interior of $P$ belongs to a component of $G \mid A$ with vertex-set $A_{1}$, say. If $u \notin A_{1}$, then $P$ can be completed to a hole by adding $u$. Since this hole is even, it follows that $P$ is even. Thus we may assume that $u \in A_{1}$. Let $A_{2}$ be the vertex-set of another component of $G \mid A$. Since every vertex of $B$ has a neighbor in $A_{2}$, there exists an induced path $P^{\prime}$ with the same ends as $P$ and with interior contained in $A_{2}$. By completing this path to a hole through the vertex $u$ we see that $P^{\prime}$ is even. Thus $P$ is even, for otherwise $P \cup P^{\prime}$ is an odd hole.

Now let $Q$ be an induced antipath of length at least two with both ends in $A$ and interior in $B_{1}$, and suppose for a contradiction that $Q$ is odd. The ends of $Q$ belong to the same component of $G \mid A$. Let the vertex-set of that component be $A_{1}^{\prime}$, and let $A_{2}^{\prime}$ be the vertex-set of another component of $G \mid A$. By (11.3) applied in the complement of $G$ to the path $\bar{Q}$ and anticonnected set $A_{2}^{\prime}$ we deduce that $\bar{Q}$ has length three. Thus $Q$ is also a path of length three, with both ends in $B_{1}$ and interior in $A$, contrary to the previous paragraph. This proves the claim.

To prove the theorem we may assume that $(A, B)$ is not rigid, and hence there exists a maximal anticonnected set $B_{1} \subseteq B$ such that the graph obtained from $G$ by adding a vertex adjacent to precisely the vertices of $B_{1}$ is not Berge. This means that there is either an induced odd path of length at least two with both ends in $B_{1}$ and interior in $A$, or an induced odd antipath of length at least two with both ends in $A$ and interior in $B_{1}$. Let $B_{2}$ be a maximal anticonnected subset of $B$ other than $B_{1}$. 
Assume first that there exists an induced path $P$ of length at least five with both ends in $B_{1}$ and interior in $A$. By applying (11.3) to $P$ and $B_{2}$ we deduce that some internal vertex of $P$ is adjacent to every vertex of $B_{2}$, and hence $(A, B)$ is rigid by the claim. Thus we may assume that no such path $P$ exists.

If $P$ is as in the previous paragraph, but of length three, then it is also an antipath with both ends in $A$ and interior in $B_{1}$. Thus it follows that $G$ has an induced odd antipath $Q_{1}$ with both ends in $A$ and interior in $B_{1}$. Let $x_{1}, y_{1} \in A$ be the ends of $Q_{1}$. Since they are adjacent, they belong to the same component of $G \mid A$. Let the vertex-set of that component be $A_{1}$, and let $A_{2}$ be the vertex-set of another component of $G \mid A$. By (11.3) applied in the complement of $G$ to the path $\overline{Q_{1}}$ and anticonnected set $A_{2}$ we deduce that $Q_{1}$ has length three (let its internal vertices be $b_{1}$ and $\left.b_{1}^{\prime}\right)$ and that there exists an induced odd path $P_{2}$ with ends $b_{1}$ and $b_{1}^{\prime}$ and all internal vertices in $A_{2}$. By the conclusion of the previous paragraph the path $P_{2}$ has length three; let its vertices be $b_{1}, x_{2}, y_{2}, b_{1}^{\prime}$, in order. By the claim and (11.3) applied to the path $P_{2}$ and anticonnected set $B_{2}$ we may assume that there exists an induced odd antipath $Q_{2}$ with ends $x_{2}, y_{2}$ and interior in $B_{2}$. The proof that $Q_{1}$ has length three applies to $Q_{2}$ as well, and so $Q_{2}$ has length three. Thus $Q_{2}$ is also a three-edge path; let its vertex set be $b_{2}, x_{2}, y_{2}, b_{2}^{\prime}$, in order. Since $Q_{1}$ cannot be completed to an odd antihole by adding $b_{2}$ or $b_{2}^{\prime}$, we deduce that each of them has a neighbor in $\left\{x_{1}, y_{1}\right\}$. But they do not have a common neighbor in $\left\{x_{1}, y_{1}\right\}$, for otherwise $Q_{2}$ can be completed to an odd antihole using that common neighbor. Thus either $b_{2}$ is adjacent to $x_{1}$ and not to $y_{1}$ and $b_{2}^{\prime}$ is adjacent to $y_{1}$ and not to $x_{1}$, or $b_{2}^{\prime}$ is adjacent to $x_{1}$ and not to $y_{1}$ and $b_{2}$ is adjacent to $y_{1}$ and not to $x_{1}$. In the former case the subgraph of $G$ induced by $\left\{x_{1}, y_{1}, x_{2}, y_{2}, b_{1}, b_{1}^{\prime}, b_{2}, b_{2}^{\prime}\right\}$ is a double diamond, and in the latter case it is isomorphic to the line graph of the graph $K_{3,3}$ with one edge deleted. In either case this is a contradiction to the fact that $G$ is bipartisan.

We remark that (11.1) comes short of proving Chvátal's skew partition conjecture in its full generality, because Chvátal conjectured the same conclusion for minimal imperfect graphs. However, (11.1) is sufficiently strong for the proof of (1.1), which in turn implies Chvátal's conjecture.

\section{Wheels}

As we pointed out earlier, it suffices to prove (8.3) for bipartisan graphs. For bipartisan graphs a stronger result holds.

12.1 Let $G$ be a bipartisan graph such that $G$ and $\bar{G}$ are not bipartite. Then $G$ has an even skew partition.

The proof of (12.1) is still fairly long, and perhaps somewhat less natural than the earlier steps. Our strategy, originally initiated by Conforti and Cornuéjols [7], is to use wheels. If $Y \subseteq V(G)$, then we say that an edge of $G$ is $Y$-complete if both its ends are complete to $Y$. A wheel in a graph $G$ is a pair $(C, Y)$, where $C$ is a hole in $G$ of length at least six and $Y \subseteq V(G)-V(C)$ is a non-empty 


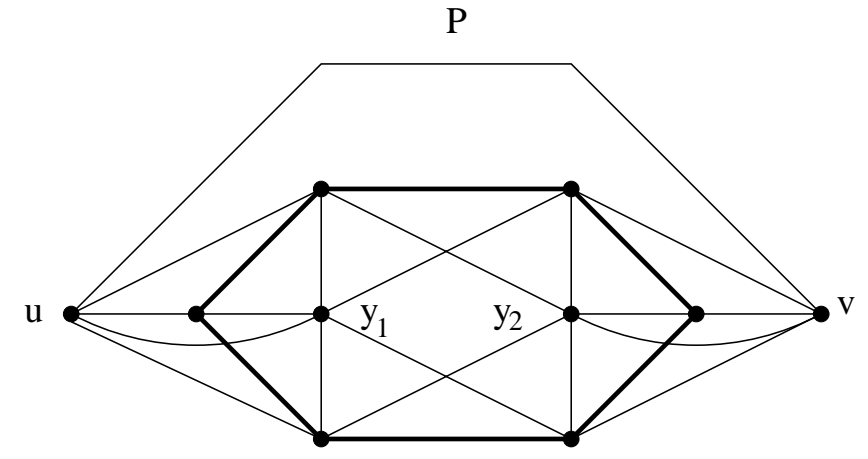

Fig. 3. A non-separable odd wheel

anticonnected set such that there are two disjoint $Y$-complete edges of $C$. We call $C$ the rim and $Y$ the hub of the wheel.

12.2 If $(C, Y)$ is a wheel in a Berge graph, then $C$ has an even number of $Y$-complete edges.

Proof. If not, then, since $C$ is even, it has an odd subpath $P$ of length at least three with both ends but no internal vertex complete to $Y$. By (11.2) applied to $P$ and $Y$ there exists either an induced odd path $R$ as in (11.2)(ii) or an induced odd antipath $Q$ as in (11.2)(iii). Since $C$ has two $Y$-complete edges, it has a vertex $z \notin V(P)$ complete to $Y$. By adding $z$ to $R$ or $Q$ as appropriate we obtain an odd hole or an odd antihole in $G$, a contradiction.

Let $(C, Y)$ be a wheel in a Berge graph $G$. We say that two vertices $u, v \in$ $V(C)$ have the same wheel parity if a subpath of $C$ joining them contains an even number of $Y$-complete edges (in which case so does the other subpath of $C$ joining them, by (12.2)), and we say they have opposite wheel parity otherwise. Now let $X$ be the set of all common neighbors of vertices of $Y$. Let us say that the wheel $(C, Y)$ is separable if some set $Z \subseteq X \cup Y$ intersects both $X$ and $Y$ and separates a vertex of one wheel parity from a vertex of the opposite wheel parity. This is a desirable property, because in this case $(V(G)-Z, Z)$ is a skew partition of $G$. Unfortunately, not all wheels are separable. First, let us point out the significance of prisms. In the example of Figure 3 the wheel $\left(C,\left\{y_{1}, y_{2}\right\}\right)(C$ is shown thick) is not separable. If the path $P$ joining the vertices $u$ and $v$ has length at least two (in fact, it must be odd), then $G$ has a prism, while if $P$ has length one, then the complement of $G$ has a prism. This example illustrates that the absence of prisms eliminates a large class of wheels that are not separable.

But even in bipartisan graphs not all wheels are separable. For instance, consider the graph in Figure 4. It is tempting to replace $C$ by the hole $v v_{1} v_{2} v_{3} v_{4} v_{5}$, but then $\{y\}$ is no longer a hub. However, this argument does work when $(C, Y)$ is an "odd wheel". A $Y$-segment is a maximal subpath of $C$ whose vertices are all complete to $Y$. We say that a wheel $(C, Y)$ is an odd wheel, if it has an odd $Y$-segment. For odd wheels the above argument indeed works: if the difficulty indicated in Figure 4 arises, then we can reroute the rim to obtain another 


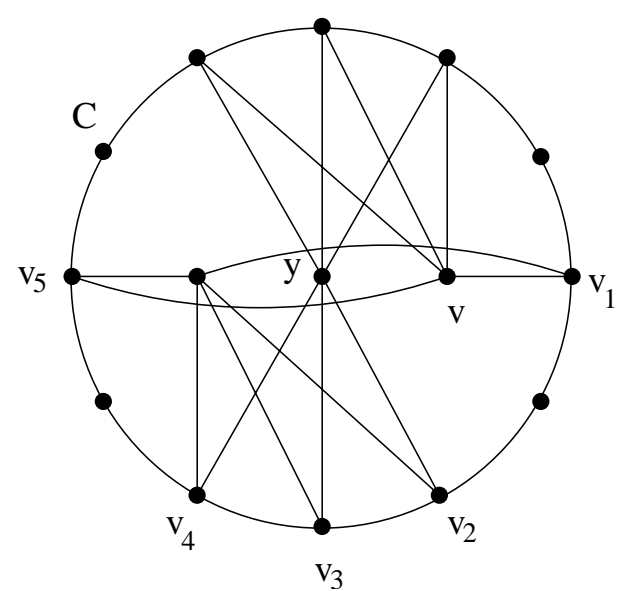

Fig. 4. A non-separable wheel in a bipartisan graph

odd wheel, which is better in a certain way. Thus we can show that some carefully selected wheel is separable, and so we have the following result, obtained independently by Conforti, Cornuéjols, Vušković and Zambelli [8].

12.3 If a bipartisan graph has an odd wheel, then it has an even skew partition.

How about wheels that are not odd? Those require a different approach. Let $(C, Y)$ be such a wheel in a bipartisan graph. Then there are three consecutive vertices $u_{1}, u_{0}, u_{2}$ on $C$, all complete to $Y$. We now find a maximal sequence $u_{1}, u_{2}, \ldots, u_{t}$ of vertices complete to $Y \cup\left\{u_{0}\right\}$ such that for all $i=3,4, \ldots, t$

- there exists a connected subset of $V(G)$ including $V(C)-\left\{u_{0}, u_{1}, u_{2}\right\}$, containing a neighbor of $u_{i}$, containing no neighbor of $u_{0}$ and containing no vertex complete to $\left\{u_{1}, u_{2}, \ldots, u_{i-1}\right\}$, and

- the vertex $u_{i}$ is not complete to $\left\{u_{1}, u_{2}, \ldots, u_{i-1}\right\}$.

Let $U=\left\{u_{1}, u_{2}, \ldots, u_{t}\right\}$, and let $V$ be the set of all $U$-complete vertices other than $u_{0}$. Now it is almost true that the set $U \cup V$ separates $\left\{u_{0}\right\}$ from $V(C)-$ $\left\{u_{0}, u_{1}, u_{2}\right\}$, in which case $(V(G)-U-V, U \cup V)$ is a skew partition. Thus we show (see [6] for details) that either $G$ has a skew partition, or $G$ has a wheel $\left(C^{\prime}, Y^{\prime}\right)$, where $Y$ is a proper subset of $Y^{\prime}$. By choosing the original wheel with $Y$ maximal we therefore obtain an extension of (12.3) to all wheels.

Finally, we must prove that for every bipartisan graph, either it satisfies the conclusion of (12.1), or either it or its complement contains a wheel. We refer to [6] for full details. A weaker result (using a less restricted definition of a wheel) was obtained independently by Conforti, Cornuéjols and Zambelli [9].

Acknowledgements. We would like to acknowledge our debt to Michele Conforti, Gérard Cornuéjols and Kristina Vušković; their pioneering work was a source of inspiration for us. Several of their ideas were seminal for this research. We would also like to acknowledge the American Institute of Mathematics, who supported two of us full-time for six months at the 
beginning of this project. Without their generous assistance our work probably would not have even started.

NR, PDS, RT were partially supported by ONR grant N00014-01-1-0608. NR was partially supported by NSF grant DMS-0071096. PDS was partially supported by ONR grant N0001497-1-0512 and NSF grant DMS-0070912. RT was partially supported by NSF grant DMS0200595 .

\section{References}

1. C. Berge, Färbung von Graphen deren sämtliche bzw. deren ungerade Kreise starr sind (Zusammenfassung), Wissenschaftliche Zeitschrift, Martin Luther Universität HalleWittenberg, Mathematisch-Naturwissenschaftliche Reihe (1961), 114-115.

2. V. Chvátal, On certain polytopes associated with graphs, J. Combin. Theory Ser. B 13 (1975), 138-154.

3. V. Chvátal, Star cutsets and perfect graphs, J. Combin. Theory Ser. B 39 (1985), 189-199.

4. V. Chvátal and N. Sbihi, Bull-free Berge graphs are perfect, Graphs and Combin. 3 (1987), 127-139.

5. M. Chudnovsky, PhD dissertation, Princeton University, 2003.

6. M. Chudnovsky, N. Robertson, P. D. Seymour and R. Thomas, The strong perfect graph theorem, manuscript, http://www.math.gatech.edu/ thomas/spgc.html.

7. M. Conforti and G. Cornuéjols, Graphs without odd holes, parachutes or proper wheels: a generalization of Meyniel graphs and of line graphs of bipartite graphs, manuscript 1999.

8. M. Conforti, G. Cornuéjols, K. Vuskovic and G. Zambelli, Decomposing Berge graphs containing proper wheels, manuscript, March 2002.

9. M. Conforti, G. Cornuéjols and G. Zambelli, Decomposing Berge graphs containing no proper wheels, stretchers or their complements, manuscript, May 2002.

10. G. Cornuéjols, The strong perfect graph conjecture, Proceedings of the ICM, Beijing, China, 2002.

11. G. Cornuéjols and W. H. Cunningham, Compositions for perfect graphs, Discrete Math. 55 (1985), 245-254.

12. I. Cziszár, J. Körner, L. Lovász, K. Marton and G. Simonyi, Entropy splitting for antiblocking corners and perfect graphs, Combinatorica 10 (1990), 27-40.

13. E. Egerváry, On combinatorial properties of matrices, Mat. Lapok 38 (1931), 16-28.

14. D. R. Fulkerson, Blocking and anti-blocking pairs of polyhedra, Math. Programming 1 (1971), 168-194.

15. D. R. Fulkerson, Anti-blocking polyhedra, J. Combin. Theory Ser. B 12 (1972), 50-71.

16. S. Gerke, Weighted colouring and channel assignment, DPhil thesis, University of Oxford, 2000.

17. S. Gerke and C. McDiarmid, Graph imperfection, J. Combin. Theory Ser. B 83 (2001), 58-78.

18. S. Gerke and C. McDiarmid, Graph imperfection II, J. Combin. Theory Ser. B 83 (2001), 79-101.

19. M. Grötschel, L. Lovász, and A. Schrijver, Geometric algorithms and combinatorial optimization, Springer-Verlag, Berlin, 1993.

20. C. T. Hoang, Some properties of minimal imperfect graphs, Discrete Math.160 (1996), $165-175$.

21. J. Kahn and J. H. Kim, Entropy and sorting, J. Comput. System Sci. 51 (1995), 390-399.

22. L. G. Khachiyan, A polynomial algorithm for linear programming (in Russian), Doklady Akademii Nauk SSSR 244 (1979), 1093-1096.

23. D. König, Über Graphen und ihre Anwendung auf Determinantentheorie und Mengenlehre, Math. Ann. 77 (1916), 453-465.

24. D. König, Graphen und Matrizen, Math. Lapok 38 (1931), 116-119.

25. J. Körner, Coding of an information source having ambiguous alphabet and the entropy of graphs, Transactions of the 6th Prague conference on information theory, Academia, Prague (1973), 411-425. 
26. L. Lovász, Normal hypergraphs and the weak perfect graph conjecture, Discrete Math. 2 (1972), 253-267.

27. L. Lovász, On the Shannon capacity of a graph, IEEE Trans. Inform. Theory 25 (1979), 1-7.

28. L. Lovász and M. Plummer, Matching theory, Annals of Discrete Math. 29, NorthHolland, Amsterdam (1986).

29. L. Lovász, Matching structure and the matching lattice, J. Combin. Theory Ser. B 43 (1987), 187-222.

30. W. McCuaig, Pólya's permanent problem, manuscript (81 pages), June 1997.

31. C. McDiarmid, Graph Imperfection and Channel Assignment, In: Perfect graphs, J. L. Ramirez Alfonsin and B. Reed Eds., Wiley, 2001.

32. C. McDiarmid and B. Reed, Channel assignment and weighted colouring, Networks 36 (2000), 114-117.

33. M. W. Padberg, Perfect zero-one matrices, Math. Programming 6 (1974), 180-196.

34. J. L. Ramírez Alfonsín and B. A. Reed, Perfect graphs, J. Wiley and Sons, Chichester, 2001.

35. B. A. Reed, A gentle introduction to semi-definite programming, In: Perfect graphs, J. L. Ramirez Alfonsin and B. Reed Eds., Wiley, 2001

36. N. Robertson, P. D. Seymour and R. Thomas, Tutte's edge-coloring conjecture, J. Combin. Theory Ser. B 70 (1997), 166-183.

37. N. Robertson, P. D. Seymour and R. Thomas, Permanents, Pfaffian orientations, and even directed circuits, Math. Ann. 150 (1999), 929-975.

38. F. Roussel and P. Rubio, About skew partitions in minimal imperfect graphs, J. Combin. Theory Ser. B 83, (2001), 171-190.

39. C. E. Shannon, The zero error capacity of a noisy channel, I.R.E. Trans. Inform. Theory IT-2 (1956), 8-19.

40. G. Simonyi, Perfect graphs and graph entropy. An updated survey, In: Perfect graphs, J. L. Ramirez Alfonsin and B. Reed Eds., Wiley, 2001.

41. A. Tucker, The strong perfect graph conjecture and an application to a municipal routing problem, Graph Theory and Applications, Lecture Notes in Math. 303, Springer, Berlin 1972.

42. A. Tucker, Perfect graphs and an application to optimizing municipal services, SIAM Rev. 15 (1973), 585-590.

43. K. Wagner, Über eine Eigenschaft der ebenen Komplexe, Math. Ann. 114 (1937), 570590 